EGU2020-11954

EGU General Assembly 2020

(c) Author(s) 2020. This work is distributed under

the Creative Commons Attribution 4.0 License.

\title{
Advanced GPR Signal Processing Techniques for Root Detection in Urban Environments
}

\author{
Livia Lantini ${ }^{1}$, Fabio Tosti ${ }^{1}$, Iraklis Giannakis ${ }^{1}$, Kevin Jagadissen Munisami ${ }^{1}$, Dale Mortimer ${ }^{2}$, and \\ Amir M. Alani ${ }^{1}$ \\ ${ }^{1}$ School of Computing and Engineering, University of West London (UWL), London, United Kingdom of Great Britain and \\ Northern Ireland (Livia.Lantini@uwl.ac.uk; Fabio.Tosti@uwl.ac.uk; Iraklis.Giannakis@uwl.ac.uk; Kevin.Munisami@uwl.ac.uk; \\ Amir.Alani@uwl.ac.uk \\ ${ }^{2}$ Tree Service, London Borough of Ealing, Perceval House, London, United Kingdom of Great Britain and Northern Ireland \\ (MortimerD@ealing.gov.uk)
}

Street trees are widely recognised to be an essential asset for the urban environment, as they bring several environmental, social and economic benefits [1]. However, the conflicting coexistence of tree root systems with the built environment, and especially with road infrastructures, is often cause of extensive damage, such as the uplifting and cracking of sidewalks and curbs, which could seriously compromise the safety of pedestrians, cyclists and drivers.

In this context, Ground Penetrating Radar (GPR) has long been proven to be an effective nondestructive testing (NDT) method for the evaluation and monitoring of road pavements. The effectiveness of this tool lies not only in its ease of use and cost-effectiveness, but also in the proven reliability of the results provided. Besides, recent studies have explored the capability of GPR in detecting and mapping tree roots [2]. Algorithms for the reconstruction of the tree root systems have been developed, and the spatial variations of root mass density have been also investigated [3].

The aim of this study is, therefore, to investigate the GPR potential in mapping the architecture of root systems in street trees. In particular, this research aims to improve upon the existing methods for detection of roots, focusing on the identification of the road pavement layers. In this way, different advanced signal processing techniques can be applied at specific sections, in order to remove reflections from the pavement layers without affecting root detection. This allows, therefore, to reduce false alarms when investigating trees with root systems developing underneath road pavements.

In this regard, data from trees of different species have been acquired and processed, using different antenna systems and survey methodologies, in an effort to investigate the impact of these parameters on the GPR overall performance.

\section{Acknowledgements}


The authors would like to express their sincere thanks and gratitude to the following trusts, charities, organisations and individuals for their generosity in supporting this project: Lord Faringdon Charitable Trust, The Schroder Foundation, Cazenove Charitable Trust, Ernest Cook Trust, Sir Henry Keswick, lan Bond, P. F. Charitable Trust, Prospect Investment Management Limited, The Adrian Swire Charitable Trust, The John Swire 1989 Charitable Trust, The Sackler Trust, The Tanlaw Foundation, and The Wyfold Charitable Trust. This paper is dedicated to the memory of our colleague and friend Jonathan West, one of the original supporters of this research project.

\section{References}

[1] J. Mullaney, T. Lucke, S. J. Trueman, 2015. "A review of benefits and challenges in growing street trees in paved urban environments," Landscape and Urban Planning, 134, 157-166.

[2] A. M. Alani, L. Lantini, 2019. "Recent advances in tree root mapping and assessment using nondestructive testing methods: a focus on ground penetrating radar," Surveys in Geophysics, 1-42.

[3] L. Lantini, F. Tosti, Giannakis, I., Egyir, D., A. Benedetto, A. M. Alani, 2019. "A Novel Processing Framework for Tree Root Mapping and Density Estimation using Ground Penetrating Radar," In 10th International Workshop on Advanced Ground Penetrating Radar, EAGE. 